Special issue of the International Conference on Computational and Experimental Science and Engineering (ICCESEN 2014)

\title{
Analytical Derivation of the Fuss Relations for Bicentric Hendecagon and Dodecagon
}

\author{
M. ORLiĆ ${ }^{a, *}$ AND Z. KALIMAN ${ }^{b}$ \\ ${ }^{a}$ University of Applied Sciences, Zagreb, Croatia \\ ${ }^{b}$ University of Rijeka, Department of Physics, Rijeka, Croatia
}

\begin{abstract}
In this article we present analytical derivation of the Fuss relations for $n=11$ (hendecagon) and $n=12$ (dodecagon). We base our derivation on the Poncelet closure theorem for bicentric polygons, which states that if a bicentric $n$-gon exists on two circles then every point on the outer circle is the vertex of same bicentric $n$-gon. We have used Wolfram Mathematica for the analytical computation. We verified results by comparison with earlier obtained results as well as by numerical calculations.
\end{abstract}

DOI: 10.12693/APhysPolA.128.B-82

PACS: $02.70 . \mathrm{Wz}$

\section{Introduction}

The bicentric $n$-gon is polygon with $n$ sides that are tangential for incircle and chordal for circumcircle. The connection between the radii of the circles and the distance of their centers represents the Fuss relation for the certain bicentric $n$-gon. The problem of finding relations between $R, r$ and $d$ (where $R$ is a radius of circumcircle, $r$ is a radius of incircle, and $d$ is distance between their centers) is considered in Ref. [1] as one of 100 great problems of elementary mathematics. Swiss mathematician Nicolaus Fuss $(1755-1826)$ was the first who has found these relations for the bicentric quadrilateral, pentagon, hexagon, heptagon and octagon. The corresponding formula for triangle was already given by Euler. Since that time, mathematicians proved the Fuss relation for these and some other bicentric polygons using various methods based on geometry, trigonometric transformation (Steiner, Jacobi, Dörrie, Chaundy, etc.), or the Jacobi elliptic function and a complete elliptic integral of the first kind (Richelot) [1]. Radić [2-5] used different methods based on elementary geometry or by introducing adequate functions in obtaining the Fuss relations for bicentric polygons which can be multiply circumscribed. Also he found connections between the Fuss relations for bicentric polygons with incircle and with ones with excircle. In Refs. [6, 7] we have given analytical derivation of the Fuss relations for pentagon and hexagon.

Here we derive the Fuss relations for hendecagon and dodecagon. Our derivations are based on the Poncelet closure theorem for bicentric polygons [8]. This theorem can be stated as follows:

Theorem 1. Let $C_{1}$ and $C_{2}$ be any given two circles in a plane such that one is completely inside of the other. Then only one of the following two assertions is true:

*corresponding author; e-mail: morlic1@tvz.hr
- There is no bicentric n-gon whose circumcircle is $C_{1}$ and incircle $C_{2}$.

- There are infinitely many bicentric n-gons whose is circumcircle $C_{1}$ and incircle $C_{2}$. For every point $A_{1}$ in $C_{1}$ there is bicentric n-gon $A_{1} \ldots A_{n}$ whose circumcircle is $C_{1}$ and incircle $C_{2}$.

We use coordinate system with $x$-axes defined by centers of the circles, and starting point of our bicentric polygon is on those axes. In actual derivation of the Fuss relations we use only upper half-plane $(y \geq 0)$ because such polygons are symmetric with respect to $x$-axes. Slightly different derivation is for odd and for even sided polygons [6], as is also described later in this article.

\section{Hendecagon}

Polygon with eleven sides is called hendecagon. The procedure is general for all odd sided bicentric polygons. Let us denote outer circle with radius $R$ by $C_{1}$, and chose coordinate system with origin in the center of $C_{1}$. Inner circle $C_{2}$ has radius $r$ and center in point $I(d, 0)$ (Fig. 1a).

Using the Poncelet closure theorem we choose first tangent $t_{1}$ on $C_{2}$ in point $P(r+d, 0)$. Tangent intersect circle $C_{1}$ in vertices $A_{1}\left(r+d, y_{1}\right)$ and $A_{11}\left(r+d, y_{11}\right)$. From vertex $A_{2}$ we get next vertex $A_{3}^{(2)}\left(x_{3}^{(2)}, y_{3}^{(2)}\right)$ where abscissa is calculated as intersection of tangent $t_{3}$ and circle $C_{1}$. Obtained expression for $x_{3}^{(2)}$ is

$$
x_{3}^{(2)}=
$$

$\overline{2\left(p^{3} q^{3}+p^{3} q^{2}-p^{3} q-p^{3}-p^{2} q^{3}+2 p^{2} q^{2}-p^{2} q-p q^{3}+p q^{2}+q^{3}\right)^{2}}$

$\times\left[p^{7} q^{6}+2 p^{7} q^{5}-p^{7} q^{4}-4 p^{7} q^{3}-p^{7} q^{2}+2 p^{7} q+p^{7}\right.$

$-p^{6} q^{7}+6 p^{6} q^{6}-p^{6} q^{5}-10 p^{6} q^{4}+5 q^{4}+5 p^{6} q^{3}+2 p^{6} q^{2}$

$-3 p^{6} q+2 p^{6}+2 p^{5} q^{7}+p^{5} q^{6}-12 p^{5} q^{5}+6 p^{5} q^{4}+6 p^{5} q^{3}$

$+p^{5} q^{2}-4 p^{5} q+p^{4} q^{7}-10 p^{4} q^{6}-6 p^{4} q^{5}+12 p^{4} q^{4}+5 p^{4} q^{3}$

$-2 p^{4} q^{2}-4 p^{3} q^{7}+5 p^{3} q^{6}+6 p^{3} q^{5}-5 p^{3} q^{4}+8 p^{3} q^{3}+p^{2} q^{7}$ 


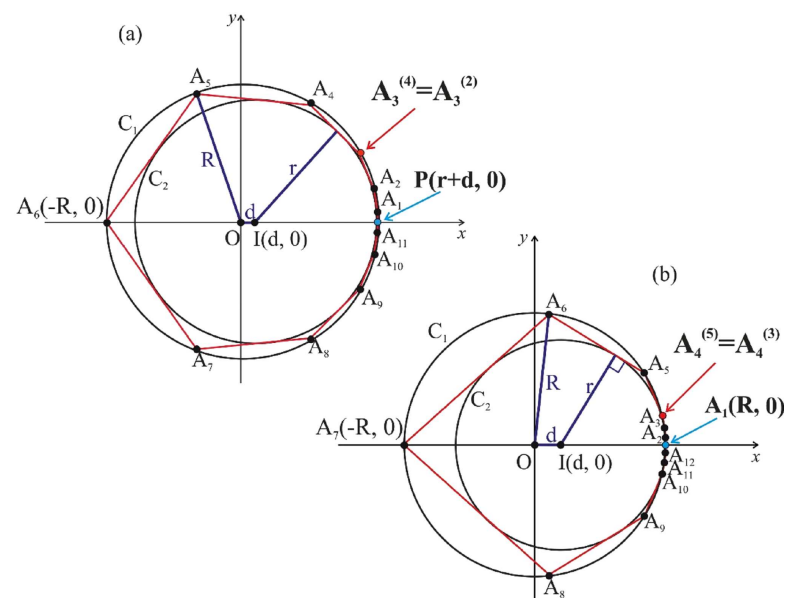

Fig. 1. (a) Symmetric bicentric hendecagon $A_{1} \ldots A_{11}$. (b) Symmetric bicentric dodecagon $A_{1} \ldots A_{12}$. They are inscribed in the circle $C_{1}$ and outscribed to $C_{2}$. Circle $C_{1}$ with the radius $R$ is centered at the origin, while $C_{2}$ is centered at $I$ with radius $r$. Distance between centers is $d$.

$\left.+2 p^{2} q^{6}-p^{2} q^{5}-2 p^{2} q^{4}+2 p q^{7}+3 p q^{6}-4 p q^{5}-q^{7}+2 q^{6}\right]$, where $p$ and $q$ are given by

$$
p=\frac{R+d}{r}, \quad q=\frac{R-d}{r} .
$$

Because of symmetry, vertex $A_{6}$ is on $x$-axes and has coordinate $(-R, 0)$. From this point we draw tangent on $C_{2}$ in the upper half-plane $t_{6}$ whose intersection with $C_{1}$ is vertex $A_{5}$. In the same way we get vertices $A_{4}$, and $A_{3}^{(4)}\left(x_{3}^{(4)}, y_{3}^{(4)}\right)$ with abscissa

$$
x_{3}^{(4)}=\frac{r(p+q)}{2 p^{2}\left(p^{4}+2 p^{2} q^{2}-2 p^{4} q^{2}-3 q^{4}+2 p^{2} q^{4}+p^{4} q^{4}\right)^{2}}
$$

$\times\left[-2 p^{8}+p^{10}+8 p^{6} q^{2}-4 p^{8} q^{2}-4 p^{10} q^{2}-12 p^{4} q^{4}+6 p^{6} q^{4}\right.$

$+6 p^{10} q^{4}+8 p^{2} q^{6}-4 p^{4} q^{6}-20 p^{6} q^{6}+20 p^{8} q^{6}-4 p^{10} q^{6}$

$\left.-2 q^{8}+p^{2} q^{8}-8 p^{4} q^{8}+22 p^{6} q^{8}-14 p^{8} q^{8}+p^{10} q^{8}\right]$.

Of course, vertices $A_{3}^{(2)}\left(x_{3}^{(2)}, y_{3}^{(2)}\right)$ and $A_{3}^{(4)}\left(x_{3}^{(4)}, y_{3}^{(4)}\right)$ are the same vertex $A_{3}$, and the coordinates must be the same. We subtract values of abscissas and equate them with zero and after inserting (2) we get

$$
(d+r+R) F_{11}(R, r, d)=0,
$$

where only factor $F_{11}(R, r, d)$ given by relation (5) can vanish and it gives the Fuss relation for hendecagon, i.e. $F_{11}(R, r, d)=0$.

$$
\begin{aligned}
& F_{11}(R, r, d)=-d^{30}-6 d^{28} r R+104 d^{26} r^{3} R-512 d^{24} r^{5} R \\
& +896 d^{22} r^{7} R-512 d^{20} r^{9} R+15 d^{28} R^{2}+12 d^{26} r^{2} R^{2} \\
& +64 d^{24} r^{4} R^{2}-192 d^{22} r^{6} R^{2}+256 d^{20} r^{8} R^{2}+84 d^{26} r R^{3} \\
& -1216 d^{24} r^{3} R^{3}+4416 d^{22} r^{5} R^{3}-4096 d^{20} r^{7} R^{3} \\
& -105 d^{26} R^{4}-156 d^{24} r^{2} R^{4}-720 d^{22} r^{4} R^{4}+320 d^{20} r^{6} R^{4}
\end{aligned}
$$

$$
\begin{aligned}
& +4608 d^{18} r^{8} R^{4}-17408 d^{16} r^{10} R^{4}+24576 d^{14} r^{12} R^{4} \\
& -16384 d^{12} r^{14} R^{4}-546 d^{24} r R^{5}+6480 d^{22} r^{3} R^{5} \\
& -16032 d^{20} r^{5} R^{5}+2304 d^{18} r^{7} R^{5}+4608 d^{16} r^{9} R^{5} \\
& -4096 d^{14} r^{11} R^{5}+8192 d^{12} r^{13} R^{5}+455 d^{24} R^{6} \\
& +936 d^{22} r^{2} R^{6}+3696 d^{20} r^{4} R^{6}+5760 d^{18} r^{6} R^{6} \\
& -34560 d^{16} r^{8} R^{6}+71680 d^{14} r^{10} R^{6}-45056 d^{12} r^{12} R^{6} \\
& +2184 d^{22} r R^{7}-20768 d^{20} r^{3} R^{7}+30080 d^{18} r^{5} R^{7} \\
& +21504 d^{16} r^{7} R^{7}-2048 d^{14} r^{9} R^{7}+24576 d^{12} r^{11} R^{7} \\
& -1365 d^{22} R^{8}-3432 d^{20} r^{2} R^{8}-11440 d^{18} r^{4} R^{8} \\
& -34560 d^{16} r^{6} R^{8}+91392 d^{14} r^{8} R^{8}-104448 d^{12} r^{10} R^{8} \\
& -6006 d^{20} r R^{9}+44440 d^{18} r^{3} R^{9}-24480 d^{16} r^{5} R^{9} \\
& -59136 d^{14} r^{7} R^{9}-19968 d^{12} r^{9} R^{9}-55296 d^{10} r^{11} R^{9} \\
& -24576 d^{8} r^{13} R^{9}-32768 d^{6} r^{15} R^{9}+3003 d^{20} R^{10} \\
& +8580 d^{18} r^{2} R^{10}+23760 d^{16} r^{4} R^{10}+94080 d^{14} r^{6} R^{10} \\
& -112896 d^{12} r^{8} R^{10}+56320 d^{10} r^{10} R^{10}+28672 d^{8} r^{12} R^{10} \\
& +16384 d^{6} r^{14} R^{10}+12012 d^{18} r R^{11}-66528 d^{16} r^{3} R^{11} \\
& -14976 d^{14} r^{5} R^{11}+64512 d^{12} r^{7} R^{11}+33792 d^{10} r^{9} R^{11} \\
& +57344 d^{8} r^{11} R^{11}+16384 d^{6} r^{13} R^{11}-5005 d^{18} R^{12} \\
& -15444 d^{16} r^{2} R^{12}-34848 d^{14} r^{4} R^{12}-153216 d^{12} r^{6} R^{12} \\
& +48384 d^{10} r^{8} R^{12}-5120 d^{8} r^{10} R^{12}-4096 d^{6} r^{12} R^{12} \\
& -18018 d^{16} r R^{13}+70752 d^{14} r^{3} R^{13}+63168 d^{12} r^{5} R^{13} \\
& -21504 d^{10} r^{7} R^{13}-12800 d^{8} r^{9} R^{13}-24576 d^{6} r^{11} R^{13} \\
& +6435 d^{16} R^{14}+20592 d^{14} r^{2} R^{14}+36960 d^{12} r^{4} R^{14} \\
& +161280 d^{10} r^{6} R^{14}+37632 d^{8} r^{8} R^{14}+12288 d^{6} r^{10} R^{14} \\
& +20592 d^{14} r R^{15}-52800 d^{12} r^{3} R^{15}-78336 d^{10} r^{5} R^{15} \\
& -21504 d^{8} r^{7} R^{15}-12288 d^{6} r^{9} R^{15}-6435 d^{14} R^{16} \\
& -20592 d^{12} r^{2} R^{16}-28512 d^{10} r^{4} R^{16}-11360 d^{8} r^{6} R^{16} \\
& -57600 d^{6} r^{8} R^{16}-20480 d^{4} r^{10} R^{16}-4096 d^{2} r^{12} R^{16} \\
& -18018 d^{12} r R^{17}+26136 d^{10} r^{3} R^{17}+54720 d^{8} r^{5} R^{17} \\
& +26496 d^{6} r^{7} R^{17}+12288 d^{4} r^{9} R^{17}+2048 d^{2} r^{11} R^{17} \\
& +5005 d^{12} R^{18}+15444 d^{10} r^{2} R^{18}+15840 d^{8} r^{4} R^{18} \\
& +48960 d^{6} r^{6} R^{18}+27648 d^{4} r^{8} R^{18}+7168 d^{2} r^{10} R^{18}
\end{aligned}
$$




$$
\begin{aligned}
& +12012 d^{10} r R^{19}-7040 d^{8} r^{3} R^{19}-22720 d^{6} r^{5} R^{19} \\
& -11264 d^{4} r^{7} R^{19}-3072 d^{2} r^{9} R^{19}-3003 d^{10} R^{20} \\
& -8580 d^{8} r^{2} R^{20}-6160 d^{6} r^{4} R^{20}-12480 d^{4} r^{6} R^{20} \\
& -4864 d^{2} r^{8} R^{20}-6006 d^{8} r R^{21}-176 d^{6} r^{3} R^{21} \\
& +5088 d^{4} r^{5} R^{21}+1792 d^{2} r^{7} R^{21}+1365 d^{8} R^{22} \\
& +3432 d^{6} r^{2} R^{22}+1584 d^{4} r^{4} R^{22}+1408 d^{2} r^{6} R^{22} \\
& +2184 d^{6} r R^{23}+864 d^{4} r^{3} R^{23}-384 d^{2} r^{5} R^{23}-455 d^{6} R^{24} \\
& -936 d^{4} r^{2} R^{24}-240 d^{2} r^{4} R^{24}-546 d^{4} r R^{25} \\
& -280 d^{2} r^{3} R^{25}-32 r^{5} R^{25}+105 d^{4} R^{26}+156 d^{2} r^{2} R^{26} \\
& +16 r^{4} R^{26}+84 d^{2} r R^{27}+32 r^{3} R^{27}-15 d^{2} R^{28} \\
& -12 r^{2} R^{28}-6 r R^{29}+R^{30}
\end{aligned}
$$

\section{Dodecagon}

Polygon with twelve sides is called dodecagon. The procedure is general for all even side bicentric polygons. Let us denote outer circle with radius $R$ by $C_{1}$ and choose coordinate system with origin in the center of $C_{1}$. Inner circle $C_{2}$ has radius $r$ and center in point $I(d, 0)$ (Fig. 1b).

For all even side bicentric polygons the starting point is $A_{1}(R, 0)$, which also represents the first vertex of polygon. The first tangent $t_{1}$ on $C_{2}$ in vertex $A_{1}$ intersect circle $C_{1}$ in vertex $A_{2}\left(x_{2}, y_{2}\right)$. By analogous procedure, we calculated vertex $A_{4}^{(3)}\left(x_{4}^{(3)}, y_{4}^{(3)}\right)$ as intersection of tangent $t_{4}$ (pulled from vertex $A_{3}$ ) and circle $C_{1}$. The abscissa of vertex $A_{4}^{(3)}$ are given by

$$
\begin{gathered}
x_{4}^{(3)}=\frac{-r(p+q)}{2 q^{2}\left(-3 p^{4}+2 p^{2} q^{2}+2 p^{4} q^{2}+q^{4}-2 p^{2} q^{4}+p^{4} q^{4}\right)^{2}} \\
\times\left[-2 p^{8}+8 p^{6} q^{2}+p^{8} q^{2}-12 p^{4} q^{4}-4 p^{6} q^{4}-8 p^{8} q^{4}+8 p^{2} q^{6}\right. \\
+8 p^{2} q+6 p^{4} q^{6}-20 p^{6} q^{6}+22 p^{8} q^{6}-2 q^{8}-4 p^{2} q^{8}+20 p^{6} q^{8} \\
\left.-14 p^{8} q^{8}+q^{10}-4 p^{2} q^{10}+6 p^{4} q^{10}-4 p^{6} q^{10}+p^{8} q^{10}\right] .
\end{gathered}
$$

Using the symmetry reason, we introduce substitution with $R$ to $-R$ in the coordinates of the vertex $A_{4}^{(3)}\left(x_{4}^{(3)}, y_{4}^{(3)}\right)$ and we get the coordinates of the vertex $A_{4}^{(5)}\left(x_{4}^{(5)}, y_{4}^{(5)}\right)$, where the abscissa is given by

$$
\begin{gathered}
x_{4}^{(5)}=\frac{(p+q) r}{2 p^{2}\left(p^{4}+2 p^{2} q^{2}-2 p^{4} q^{2}-3 q^{4}+2 p^{2} q^{4}+p^{4} q^{4}\right)^{2}} \\
\times\left[-2 p^{8}+p^{10}+8 p^{6} q^{2}-4 p^{8} q^{2}-4 p^{10} q^{2}-6 p^{6} q^{4}+6 p^{10} q^{4}\right. \\
+8 p^{2} q^{6}-4 p^{4} q^{6}-20 p^{6} q^{6}+20 p^{8} q^{6}-4 p^{10} q^{6}-2 q^{8}+p^{2} q^{8} \\
\left.-8 p^{4} q^{8}+22 p^{6} q^{8}-14 p^{8} q^{8}+p^{10} q^{8}\right] .
\end{gathered}
$$

The vertices $A_{4}^{(3)}\left(x_{4}^{(3)}, y_{4}^{(3)}\right)$ and $A_{4}^{(5)}\left(x_{4}^{(5)}, y_{4}^{(5)}\right)$ are the same, i.e. must have the same coordinates. We subtract values of abscissas and equate them with zero and after inserting (2) we get

$-2 R\left(d^{4}-2 d^{2} r^{2}-2 d^{2} R^{2}-2 r^{2} R^{2}+R^{4}\right) F_{12}(R, r, d)=0$

where factor $F_{12}(R, r, d)$ is given as

$$
\begin{aligned}
& F_{12}(R, r, d)=d^{32}-16 d^{30} r^{2}+16 d^{28} r^{4}-16 d^{30} R^{2} \\
& +208 d^{28} r^{2} R^{2}+224 d^{26} r^{4} R^{2}-1792 d^{24} r^{6} R^{2} \\
& +5888 d^{22} r^{8} R^{2}-8192 d^{20} r^{10} R^{2}+4096 d^{18} r^{12} R^{2} \\
& +120 d^{28} R^{4}-1232 d^{26} r^{2} R^{4}-3920 d^{24} r^{4} R^{4} \\
& +16128 d^{22} r^{6} R^{4}-40960 d^{20} r^{8} R^{4}+28672 d^{18} r^{10} R^{4} \\
& -4096 d^{16} r^{12} R^{4}-560 d^{26} R^{6}+4368 d^{24} r^{2} R^{6} \\
& +23744 d^{22} r^{4} R^{6}-62720 d^{20} r^{6} R^{6}+121600 d^{18} r^{8} R^{6} \\
& +20480 d^{16} r^{10} R^{6}+57344 d^{14} r^{12} R^{6}-65536 d 12 r^{14} R^{6} \\
& + \\
& + \\
& + \\
& + \\
& + \\
& +
\end{aligned}
$$




$$
\begin{aligned}
& -3920 d^{4} r^{4} R^{24}-1792 d^{2} r^{6} R^{24}-560 d^{6} R^{26} \\
& -1232 d^{4} r^{2} R^{26}+224 d^{2} r^{4} R^{26}+120 d^{4} R^{28} \\
& +208 d^{2} r^{2} R^{28}+16 r^{4} R^{28}-16 d^{2} R^{30} \\
& -16 r^{2} R^{30}+R^{32}
\end{aligned}
$$

can vanish and it gives the Fuss relation for dodecagon i.e. $F_{12}(R, r, d)=0$.

We got required relations for hendecagon and dodecagon by subtracting values of abscissas of appropriate vertices. Also we got the same relation by subtracting values of ordinates of same vertices.

\section{Conclusion}

Throughout history mathematicians proved the Fuss relations for bicentric polygons using different methods based on geometry, trigonometric transformations or the Jacobi elliptic function and a complete elliptic integral of the first kind. Until now we have derived the Fuss relations using analytical geometry for $n$ from 4 to 12 by method described for hendecagon and dodecagon in this article. Our goal is analytical derivation of the Fuss relation for $n=13$.

\section{Acknowledgments}

This work was supported by the University of Rijeka, project number 13.12.1.4.07.

\section{References}

[1] H. Dörrie, 100 Great Problems of Elementary Mathematics, Dover, New York 1965.

[2] M. Radić, Z. Kaliman, Rad HAZU, Matematičke znanosti 503, 21 (2009) (in Croatian).

[3] M. Radić, Rad HAZU, Matematičke znanosti 519, 145 (2014) (in Croatian).

[4] M. Radić, Math. Commun. 19, 139 (2014).

[5] M. Radić, Compt. Rend. Math. 348, 415 (2010).

[6] Z. Kaliman, M. Orlić, in: 6 Int. Conf. Aplimat 2007, Vol. 3, 2007, p. .

[7] M. Orlić, Z. Kaliman, N. Orlić, in: 6 Int. Conf. Aplimat 200\%, Vol. 3, 2007, p. 83.

[8] http://mathworld.wolfram.com/PonceletsPorism.html. 